\title{
CHEMICAL MODIFICATIONS OF THE CAPSID FOR REDIRECTING AND IMPROVING THE EFFICACY OF AAV VECTORS
}

\author{
Anh K. Lam ${ }^{1}$, Dylan Frabutt ${ }^{1}$, Lei $\mathrm{Li}^{2}$, and Weidong Xiao ${ }^{1}$ \\ ${ }^{1}$ Department of Pediatrics, Herman B Wells Center for Pediatrics Research, Indiana \\ University School of Medicine, Indianapolis, IN, USA \\ ${ }^{2}$ Department of Chemistry, Georgia State University, Atlanta, GA, USA \\ Keywords: Adeno-associated virus, AAV, chemical modification, capsid engineering, capsid \\ modification.
}


Abstract

Adeno-associated virus (AAV) vector directed Gene therapy is one of the most exciting modalities of biotechnology as more applications enter clinical stage. Although $A A V$ vectors generally feature low toxicity, high stability, and long-lasting transgene expression, potential challenging issues of AAV include high vector dose, limited tissue tropism, and the host immune response and inflammation, which are all related to the capsid protein. To overcome these challenges, various strategies have been developed to engineer AAV capsids. Apart from widely employed genetic engineering of capsid protein, powerful and versatile chemical modification strategies are underexploited. This minireview summarizes recent advances and our perspectives for future direction in AAV capsid chemical modification to enhance its therapeutic use for gene therapy. 
Introduction

Over the past three decades, recombinant adeno-associated virus (rAAV) vectors have been proven to be successful tools for gene therapy against many genetic diseases. Wild-type AAVs are icosahedral single-stranded (ss) DNA parvoviruses with around 4.7 kilobase (kb) genome. ${ }^{1,2}$ They are non-pathogenic and contain non-enveloped protein capsid. rAAV vectors have been used to conduct over 200 clinical trials since 1989, with several achieving FDA-approval. ${ }^{3}$ WT AAV's genome consists of 3 open reading frames: rep, cap and $\mathrm{X}$ genes. Rep encodes for four proteins for regulation, replication, and assembly. Cap encodes for three overlapping capsid proteins: VP1 (90 kDa), VP2 (72 kDa), and VP3 $(60 \mathrm{kDa})$, with an abundance ratio of 1:1:10. Within an alternate reading frame of cap, AAP (assembly-activating protein) gene is also known to help with the capsid assembly. $X$ gene is responsible for encoding replication supportive proteins. ${ }^{4}$ Viral capsid sequence is responsible for attachment to host cell receptors and subsequent internalization, which determines vector tropism. ${ }^{5}$ Due to the requirement of high viral titers for productive transduction of the target tissue, the therapeutic index of AAV vectors is still relatively low compared to adenovirus or lentiviral-based gene therapy vectors. Their broad tissue tropism also provides an incentive for the development of vectors that can target rAAV to a specific tissue for more efficient transduction, which would reduce the titer of virus required for a therapy and also reduce adverse side effects caused by capsid immunogenicity. While progress is underway, strategies for capsid modification to improve the clinical efficacy of AAV vectors have shown with strong potentials.

\section{Genetic modification of AAV's capsid}

A comprehensive review by Büning and Srivastava summarizes genetic approaches on AAV capsid modification. ${ }^{6}$ As a rational approach, incorporation of known ligands can be performed in packaging cell lines using genetic means as a VP2-fusion protein or using biochemical means like split intein-mediated protein trans-splicing. ${ }^{7}$ These methods can be disruptive for viral packaging, which may require further optimization for the successful production of the vector in relevant quantities for laboratory or clinical studies. Directed evolution approaches utilize AAV vectors that code for their own rep and cap and apply a selection pressure in the growth environment. Combinations of rational design and 
directed evolution have also been performed, where a feature such as the binding sites of neutralizing antibodies against AAV8 can be identified, randomized, and then selected for packaging and transduction. ${ }^{8}$ Selection screens of AAV peptide display, error-prone PCR, or DNA shuffling library can also be a high-throughput approach for identifying targeting ligands (Figure 1). Genetic modifications of the AAV capsid allows for selections to be performed and the amino acid sequence to get determined from the vector DNA. However, the process is often time consuming. Selecting for tissue tropism specificity with genetic engineering also comes at the expense of packaging efficiency and short-term or non-reproducible vector infectivity. ${ }^{1,3,6}$

\section{Protein Chemical Modification}

Among many approaches that have been developed to improve viral vectors in gene therapy, chemical modification has recently been exploited and gained a promising role. Chemical modification of proteins is an important approach in research and development of new and improved products from food, agriculture, to medicine. Proteins are predominantly modified through amino acids with the most reactive side chains. These modifications often rely on the chemical reactions of oxidation/reduction, and nucleophilic/electrophilic substitutions, which is well summarized previously. ${ }^{9}$ Specifically, the thiol group found on the amino acid, cysteine (Cys), is the most reactive target for protein chemical modification, followed by the primary amino functionality found on side chain of lysine (Lys). The average abundance of Cys residues ( 3\%) on proteins is less than that of Lys ( 7\%), which enables relatively site-specific modification or chemical labeling using thiol-reactive probes. ${ }^{10}$ The unprotonated amino groups of lysine are nucleophilic, which makes lysine the next key target for chemical modification. Depending on the availability of such functional groups exposed on a protein as well as its binding activity, either Cys or Lys residues can be targeted for specific modification. Some common chemical labeling reactions are shown in Figure 2. The conjugation addition of thiol groups can be carried out using Michael acceptors such as maleimides or with alkyl halides and iodoacetamides to form thioethers (Figure 2a). Maleimides and iodoacetamides can react with the free base form of amino groups (such as Lys) if the condition is above $\mathrm{pH} 8 .{ }^{11}$ The 
primary amines can react with isothiocyanates to form thioureas, or with $\mathrm{N}$-hydroxyl succinimide (NHS) esters to form carboxamides with stable amide bonds (Figure 2b). ${ }^{12}$

Additionally, tyrosine (Tyr) residues can be modified by either at the hydroxyl group or by aromatic ring substitution. One common method is esterification of the hydroxyl group, but hydroxyl modifications are often reversible during reagent removal. The guanidinium group of arginine ( $\mathrm{Arg}$ ) can react with $\alpha$-dicarbonyl compounds such as methylglyoxal (Figure 2c) to form hydroimidazolone products. ${ }^{13,14}$ Other functional groups like the indole of tryptophan (Trp) and imidazole of histidine (His) can also be modified using substitutions and alkylations or nucleophilic attacks. ${ }^{10}$ Epoxides are known for efficient labeling of histidine in alkaline condition (Figure 2d). However, the reagents or conditions that are required to modify these functional groups are also reactive towards the thiol or amino groups of Cys and Lys residues, making the chemo-selectivity challenging. ${ }^{15}$

There are 60 monomeric proteins forming the icosahedral capsid that encases AAV genetic information and determining the tropism of the viral vector. The capsid can be chemically tailored to manipulate its therapeutic function. However, each rAAV serotype contains different capsid peptide sequences exposing unique amino acids. These exposed amino acids on the capsids are keys for selective chemical modifications. A minor change or addition to surface amino acids can ultimately alter the receptor binding motif of the vector or how it interacts with the host's immune response. However, a knowledge gap regarding the interaction of $A A V$ capsid sequences and their binding receptors adds more challenges for chemical modification of AAV. The need for investigations of AAV's chemical modification is clearly unmet. This minireview focuses on current studies regarding rAAV capsid chemical engineering to enhance its therapeutic use in gene therapy.

\section{AAV Capsid Chemical Modifications}

\section{Non-selective PEGylation on Lys}

Polymeric chemical moieties have long been utilized by crosslinking to the surface of viral vectors, which can act as a shield to evade neutralizing antibodies. They are covalently attached to the viral capsid using chemical reactivity of nucleophilic groups of 
exposed amino acids such as Lys and Cys residues. Conjugated addition of polyethylene glycol (PEG) has extensively been carried out on adenoviruses to reduce immune response and extend circulatory half-life. ${ }^{16-19}$ Lee et al used biotin-PEG-NHS with functional group of electrophilic succinimidyl propionic acid (SPA) to crosslink with Lys residues on AAV2 capsid. They examined 3 molecular weights of PEG-2000, -5000 , and -20000 Da. Biotin acts as a label for the detection of PEGylated AAVs using horseradish peroxidase conjugated streptavidin via Western blotting. For each PEG molecular weight, the molar ratio of PEG:Lys also vary for systemic comparison in transduction efficiency and antibody neutralization. All three polymer sizes didn't enhance the infectivity of the AAV virus, in fact, infectivity decreased significantly at high molar ratio of PEG:Lys. However, protection of AAV2 by PEG-2000 (molar ratio of 1000: 1) from serum neutralization moderately increased $\sim 2.3$ folds. Modification with a larger PEG-20000 alter AAV morphology significantly, and was proposed to block the cell receptor binding hence leading to low infectivity of the virus. ${ }^{20}$

\section{$\underline{\text { Non-selective small molecule modification on Arg and Lys }}$}

Without any peptide insertions, Horowitz et al chemically glycated exposed arginine (Arg) residues on the capsid of AAV2 (which clustered around the primary receptor heparan sulfate) by using methylglyoxal. Methylglyoxal (MGO) is a $\alpha$-dicarbonyl compound that is known to be highly reactive to form covalent adducts with Arg (chemical reaction as in Figure $1 \mathrm{~d}$ ) and Lys residues. Glycated AAV2 was found to be significantly lower in heparin binding as well as $>2$-fold decrease in binding to monoclonal antibody A20. However, transduction efficiency was lowered 1000-fold compared to unmodified AAV2. Intravenous tail vein injection of glycated AAV2 into mice showed expanded viral tissue tropism compared to unmodified AAV2. Interestingly, the transgene expression of luciferase found in cardiac and skeletal muscles was 100 -fold more by glycated AAV2 than by AAV2 vectors. These results highlighted the feasibility of altering AAV morphology and tropism by simple chemical modification of AAV capsid. ${ }^{13}$ Pearce et al also glycate the Arg residues on the capsid of AAV6 with 4-azidophenyl glyoxal (APGO). This APGO was then further covalently conjugated with a single-chain antibody (scFv) that is actively targeting inflamed endothelia through vascular cell adhesion molecule (VCAM-1). With optimized 
modification, the natural tropism of WT AAV6 was removed, and its transduction of endothelial cells was significantly enhanced about 4-5 folds in vitro, highlighting the feasibility of retargeting AAV to a specific host cell by chemical modification of the capsid. $^{21}$

In a different study, Mevel et al exploited surface Lys residues on the AAV capsid to react with amine-reactive molecules. Proof of concept was done using a fluorophore FITC (fluorescein isothiocyanate) and a control of fluorescein without the reactive isothiocyanate group (Figure 3). Dot blot and Western blot analyses were performed to confirm the chemical reaction using either anti-AAV antibody to recognize the capsid, antiFITC antibody, or direct fluorescence emission. Buffer TBS at pH 9.3 was chosen for labeling the primary amine which does not affect the AAV infectivity. The density of FITC molecules on the capsid was also modulated to fine-tune the viral therapeutic index. Using this concept, $\mathrm{N}$-acetylgalactosamine (GalNAc) -a monosaccharide ligand that binds to a highly expressed receptor of hepatocytes-was coupled with the reactive isothiocyanate group (-NCS). The GaINAc-NCS molecule was chemically attached to AAV8 capsid and was shown, in vivo, to reduce serotype-specific neutralizing antibodies. While significant increase in transduction efficiency was found for GaINAc-AAV at ten days post administration on mice, no difference showed at 21 days, when compared to unmodified virus. This work highlights a promising therapeutic advancement by using chemical modification on AAV capsid for redirecting the tropism as well as enhancing the transduction efficiency. ${ }^{22}$

\section{Site-specific modification via peptide insertion}

For a more site-specific modification, Liu et al implemented a genetic insertion of 13-amino-acid (including Cys) sequence into the cap gene of AAV2 (amino acid 587 within VP1/VP2/VP3 regions). Then inserted Cys was going under chemical modification to convert into an aldehyde tag. This aldehyde tag was further chemically modified with covalent conjugation of hydrazide- or hydroxylamine-functional fluorophore such as Alexahydrazide. Figure 4 shows the schematic flow of their approach. Monoclonal A20 antibody which is specific for intact AAV2 was subsequently used to confirm the chemical reaction of the viral vector and the aldehyde tag by overlaid imaging. ${ }^{23}$ To examine the transduction 
efficiency of this method, AAV-aldehyde-tag was conjugated with a monoclonal antibody against human leukocyte antigen (anti-HLA). HepG2 cells have limited permissiveness to wildtype AAV2, but anti-HLA-AAV2 expanded this tropism and significantly increased the transduction from $\sim 2 \%$ to $\sim 12 \%$. This study indicated an active targeting approach for expanding AAV tropism using chemically-modified AAV capsid bearing specific ligands, which could be a promise for cancer gene therapy. ${ }^{23}$ In a similar concept, Chandran et al inserted a tetracysteine-peptide into the capsid (amino acid 138 of VP1 and VP2) of AAV9 without compromising the viral infectivity. ${ }^{24}$ The functional thiol groups of this tetracysteine allowed for further incorporation of a fluorescent dye to the capsid through a thiol-maleimide reaction. Fluorescent-labeled viruses enabled monitoring of the modified particles in vivo, leading to the generation of two capsid interactomes: blocking integrin $\alpha \mathrm{V} \beta 6$ receptor decreased AAV9 transduction, and lower expression of histone deacetylase 4 (HDAC4) increased its transduction.

\section{Site-specific modification via unnatural amino acid (UAA) incorporation}

Unnatural amino acids (UAA) bearing bioorthogonal chemical groups (e.g., azide group) can be incorporated into the AAV capsid during transfection to produce taggedAAV particles for subsequent chemical modifications via specific crosslinking chemistries (Figure 5). Specifically, Zhang et al expanded the genetic code of AAV2 by adding a plasmid vector pAAV-RC-TAG (TAG, stop codon at different sites on the cap gene) and a UAA N-2azideoethyloxycarbonyl-L-lysine to a typical triple-plasmid transfection. ${ }^{25}$ This step allowed them to harvest azide-AAV2, and the azide functional group allowed for further bioorthogonal conjugation of cRGD (cyclic tri-peptide of Arg-Gly-Asp that is known as a ligand of integrin avB3 molecules highly expressed in a wide variety of tumors) via azidealkyne Huisgen cycloaddition in a site-specific manner. AAV2-N587+1/UAA/RGD was chosen for in vivo study since it best enhanced the transduction of the suicide agent Herpes simplex virus thymidine kinase (KT) which is most widely used in cancer gene therapy in response to the prodrug ganciclovir. At day 15 , this modified AAV2$\mathrm{N} 587+1 /$ UAA/RGD significantly suppressed the tumor volume in rats by $\sim 8$ folds compared to the WT AAV2, and $\sim 3.5$ folds compared to the AAV2-N587RGD (mutation without using UAA). 
The same concept of altering the tropism of AAV2 by incorporating azido-modified UAA to the capsid site R588 for attaching the CRGD motif was also investigated by Kelemen et al. ${ }^{26}$ In vitro infectivity of AAV2-R588/UAA/CRGD was $20 \%$ lower than the WT control on the ovarian tumor-derived cells and completely lost on HEK293 cells. ${ }^{25}$ Through a similar concept, Yao and Zhou et al modified AAV2 with azido-modified UAA at the capsid sites Q325+1, S452+1, and R585+1, for a subsequent site-selective PEGylation (20kD PEG) into these azide groups. Viral titers of these modified viruses were several folds lowered than the WT control but their protection against antibody recognition in rats was moderately improved ( 20\%). However, the transduction of these modified viruses in vivo was not discussed. ${ }^{27}$ In a different study, Katrekar et al engineered AAV2 and AAV-DJ by combining the insertion of UAA with a reactive group and further chemical conjugation of that group. Four surface capsid residues R447, S578, N587, and S662 were co-translationally substituted with an azide modified pyrrolysine derivative, which again allow specific chemical conjugation via azide-alkyne Huisgen cycloaddition. ${ }^{28}$ Since AAV-DJ is a chimera of AAV serotypes 2,8 , and 9, its titer and infectivity outperform AAV2 about 10 folds in multiple cell lines and naive mice. Mutant AAV-DJ-N589UAA produced a titer 15-fold higher than AAV2-N587UAA; both mutants did not alter the virus infectivity. Later azidoalkyne click chemistry added an oligonucleotide of $10 \mathrm{kDa}$, which could be conjugated with multi-functionality probe such as biotin. Conjugated biotin was used to confirm the UAA modified AAV. The oligo-AAV was masked with lipofectamine as a shield that protected the virus from neutralizing antibodies. One limitation is that the UAA modified capsid lead to several folds lower in viral titer. ${ }^{28}$ These studies show the successful incorporation of a UAA insert into the AAV capsid and using specific chemistry of the insert to tether the AAV capsid to a spectrum of different molecules for different purposes.

\section{Conclusion}

The rising knowledge of AAV biology has enabled more directed design of chemical modification for AAV capsid. Whether to protect the virus from neutralizing antibodies or to redirect its tropism to specific tissues, chemical modification is undoubtedly capable of. Future directions are not limited there, as chemical engineering can also tailor the capsid to be more controllable therapeutic agent. For instance, capsid was tethered with a 
fluorophore for in vivo imaging monitor ${ }^{24}$ or to be altered the capsid geometry for enhancing its stability. ${ }^{29}$ Although more and more AAV gene therapy drugs have entered clinical trials, challenges exist. One of them is the lack of a standard system for testing the efficacy and safety of AAV vectors on humans. Engineered AAV vectors could show great potentials in animal models or even humanized animals but might not transfer to other kinds of animals and humans. If AAV capsid can be engineered so that it becomes more specific tropism for a targeted tissue, its required doses would be reduced, leading to greater transduction efficiency and lower unwanted immunogenicity. Although investigations on $A A V$ chemical modification are underexploited, the rate of novel innovation in gene and cell therapy will quickly produce more and more promising engineered candidates. As more and more complete structures of wild-type AAV capsids have been discovered and published, better and clearer strategies in chemical modification can be implemented and developed. Efforts are underway with one common goal-to benefit patients. 
References

1. Grimm, D., and Zolotukhin, S. (2015). E Pluribus Unum: 50 Years of Research, Millions of Viruses, and One Goal--Tailored Acceleration of AAV Evolution. Mol Ther 23, 1819-1831. 10.1038/mt.2015.173.

2. Xiao, W., Chirmule, N., Berta, S.C., McCullough, B., Gao, G., and Wilson, J.M. (1999). Gene therapy vectors based on adeno-associated virus type 1. Journal of virology 73, 3994-4003.

3. Li, C., and Samulski, R.J. (2020). Engineering adeno-associated virus vectors for gene therapy. Nat Rev Genet 21, 255-272. 10.1038/s41576-019-0205-4.

4. Cao, M., You, H., and Hermonat, P.L. (2014). The X gene of adeno-associated virus 2 (AAV2) is involved in viral DNA replication. PLoS One 9, e104596.

5. Zincarelli, C., Soltys, S., Rengo, G., and Rabinowitz, J.E. (2008). Analysis of AAV serotypes 1-9 mediated gene expression and tropism in mice after systemic injection. Mol Ther 16, 1073-1080. 10.1038/mt.2008.76.

6. Buning, H., and Srivastava, A. (2019). Capsid Modifications for Targeting and Improving the Efficacy of AAV Vectors. Mol Ther Methods Clin Dev 12, 248-265. 10.1016/j.omtm.2019.01.008.

7. Reul, J., Muik, A., and Buchholz, C.J. (2019). Ligand coupling to the AAV capsid for cell-specific gene transfer. In Adeno-Associated Virus Vectors, (Springer), pp. 35-50.

8. Havlik, L.P., Simon, K.E., Smith, J.K., Klinc, K.A., Tse, L.V., Oh, D.K., Fanous, M.M., Meganck, R.M., Mietzsch, M., Kleinschmidt, J., Agbandje-McKenna, M., et al. (2020). Coevolution of Adeno-associated Virus Capsid Antigenicity and Tropism through a Structure-Guided Approach. J Virol 94. 10.1128/JVI.00976-20.

9. Boutureira, O., and Bernardes, G.J. (2015). Advances in chemical protein modification. Chem Rev 115, 2174-2195. 10.1021/cr500399p.

10. FEENEY, R.E., YAMASAKI, R.B., and GEOGHEGAN, K.F. (1982). Chemical modification of proteins: an overview.

11. Chalker, J.M., Bernardes, G.J., Lin, Y.A., and Davis, B.G. (2009). Chemical modification of proteins at cysteine: opportunities in chemistry and biology. Chem Asian J 4, 630-640. 10.1002/asia.200800427. 
12. Basle, E., Joubert, N., and Pucheault, M. (2010). Protein chemical modification on endogenous amino acids. Chem Biol 17, 213-227. 10.1016/j.chembiol.2010.02.008.

13. Horowitz, E.D., Weinberg, M.S., and Asokan, A. (2011). Glycated AAV vectors: chemical redirection of viral tissue tropism. Bioconjug Chem 22, 529-532. $10.1021 / \mathrm{bc} 100477 \mathrm{~g}$.

14. Lo, T., Westwood, M.E., McLellan, A.C., Selwood, T., and Thornalley, P.J. (1994). Binding and modification of proteins by methylglyoxal under physiological conditions. A kinetic and mechanistic study with $\mathrm{N}$ alpha-acetylarginine, $\mathrm{N}$ alphaacetylcysteine, and $\mathrm{N}$ alpha-acetyllysine, and bovine serum albumin. Journal of Biological Chemistry 269, 32299-32305.

15. Reddy, N.C., Kumar, M., Molla, R., and Rai, V. (2020). Chemical methods for modification of proteins. Org Biomol Chem 18, 4669-4691. 10.1039/d0ob00857e.

16. Croyle, M., Le, H., Linse, K., Cerullo, V., Toietta, G., Beaudet, A., and Pastore, L. (2005). PEGylated helper-dependent adenoviral vectors: highly efficient vectors with an enhanced safety profile. Gene therapy $12,579-587$.

17. Hofherr, S.E., Mok, H., Gushiken, F.C., Lopez, J.A., and Barry, M.A. (2007). Polyethylene glycol modification of adenovirus reduces platelet activation, endothelial cell activation, and thrombocytopenia. Human gene therapy 18, 837848.

18. O'Riordan, C.R., Lachapelle, A., Delgado, C., Parkes, V., Wadsworth, S.C., Smith, A.E., and Francis, G.E. (1999). PEGylation of adenovirus with retention of infectivity and protection from neutralizing antibody in vitro and in vivo. Human gene therapy 10 , 1349-1358.

19. Espenlaub, S., Wortmann, A., Engler, T., Corjon, S., Kochanek, S., and Kreppel, F. (2008). Reductive amination as a strategy to reduce adenovirus vector promiscuity by chemical capsid modification with large polysaccharides. The Journal of Gene Medicine: A cross-disciplinary journal for research on the science of gene transfer and its clinical applications 10, 1303-1314.

20. Lee, G.K., Maheshri, N., Kaspar, B., and Schaffer, D.V. (2005). PEG conjugation moderately protects adeno-associated viral vectors against antibody neutralization. Biotechnol Bioeng 92, 24-34. 10.1002/bit.20562. 
21. Pearce, H.A., Qian, H., Connell, T.U., Huang, D., Gottstein, C., Donnelly, P.S., Peter, K., Gregorevic, P., and Hagemeyer, C.E. (2019). Site-Specific Glycation and Chemoenzymatic Antibody Sortagging for the Retargeting of rAAV6 to Inflamed Endothelium. Mol Ther Methods Clin Dev 14, 261-269. 10.1016/j.omtm.2019.07.003.

22. Mével, M., Bouzelha, M., Leray, A., Pacouret, S., Guilbaud, M., Penaud-Budloo, M., Alvarez-Dorta, D., Dubreil, L., Gouin, S.G., Combal, J.P., Hommel, M., et al. (2020). Chemical modification of the adeno-associated virus capsid to improve gene delivery. Chemical Science 11, 1122-1131. 10.1039/c9sc04189c.

23. Liu, Y., Fang, Y., Zhou, Y., Zandi, E., Lee, C.L., Joo, K.I., and Wang, P. (2013). Sitespecific modification of adeno-associated viruses via a genetically engineered aldehyde tag. Small 9, 421-429. 10.1002/smll.201201661.

24. Chandran, J.S., Sharp, P.S., Karyka, E., Aves-Cruzeiro, J., Coldicott, I., Castelli, L., Hautbergue, G., Collins, M.O., and Azzouz, M. (2017). Site Specific Modification of Adeno-Associated Virus Enables Both Fluorescent Imaging of Viral Particles and Characterization of the Capsid Interactome. Sci Rep 7, 14766. 10.1038/s41598-017$15255-2$

25. Zhang, C., Yao, T., Zheng, Y., Li, Z., Zhang, Q., Zhang, L., and Zhou, D. (2016). Development of next generation adeno-associated viral vectors capable of selective tropism and efficient gene delivery. Biomaterials 80, 134-145. 10.1016/j.biomaterials.2015.11.066

26. Kelemen, R.E., Mukherjee, R., Cao, X., Erickson, S.B., Zheng, Y., and Chatterjee, A. (2016). A Precise Chemical Strategy To Alter the Receptor Specificity of the AdenoAssociated Virus. Angew Chem Int Ed Engl 55, 10645-10649. 10.1002/anie.201604067.

27. Yao, T., Zhou, X., Zhang, C., Yu, X., Tian, Z., Zhang, L., and Zhou, D. (2017). SiteSpecific PEGylated Adeno-Associated Viruses with Increased Serum Stability and Reduced Immunogenicity. Molecules 22. 10.3390/molecules22071155.

28. Katrekar, D., Moreno, A.M., Chen, G., Worlikar, A., and Mali, P. (2018). Oligonucleotide conjugated multi-functional adeno-associated viruses. Sci Rep 8, 3589. 10.1038/s41598-018-21742-x. 
29. Stone, N.P., Demo, G., Agnello, E., and Kelch, B.A. (2019). Principles for enhancing virus capsid capacity and stability from a thermophilic virus capsid structure. Nature communications $10,1-13$. 
Figures

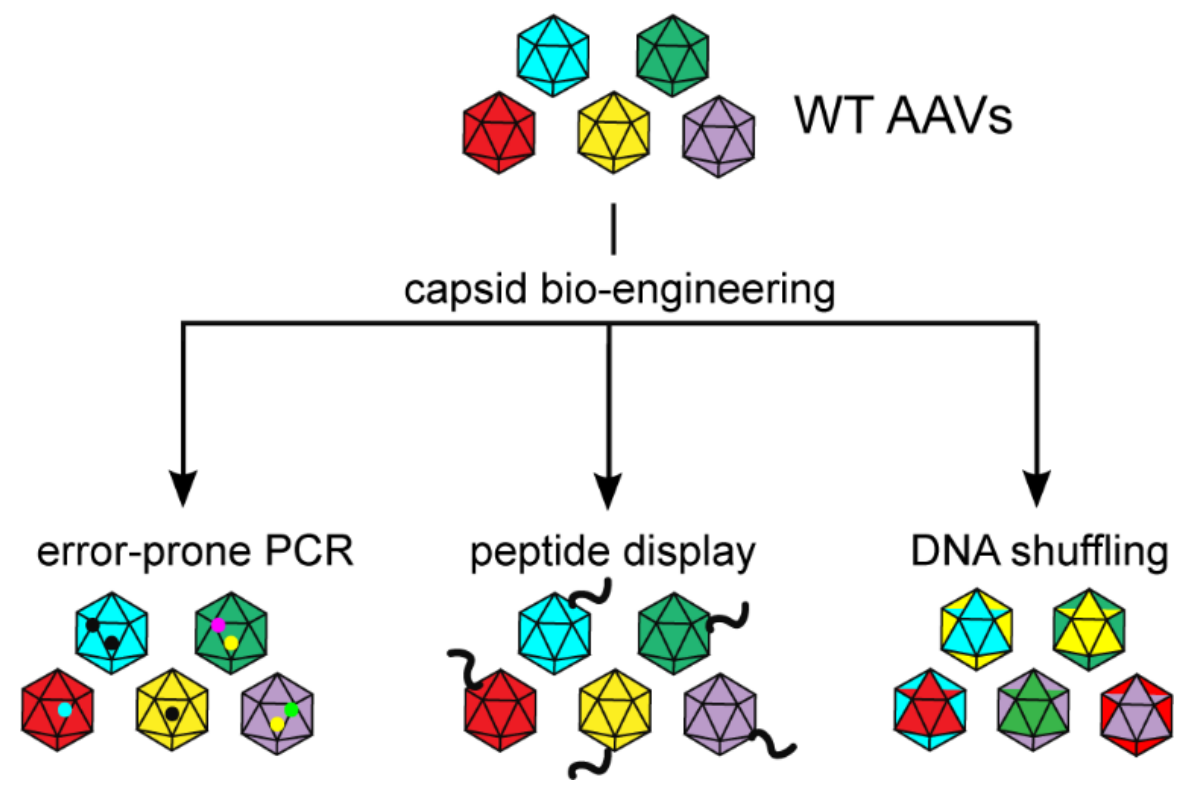

Figure 1. Summary of genetic modification approaches for AAV capsids. Generation of highly diverse mutants from either error-prone PCR, peptide display library, or DNA shuffling among different capsid serotypes offer in vivo selection screenings. Enhanced AAV transduction and/or evasion of neutralizing antibodies can be achieved through these approaches. 
a.<smiles>[R]CCCC1C=C(CC)C(=O)N1[R]</smiles>

b.

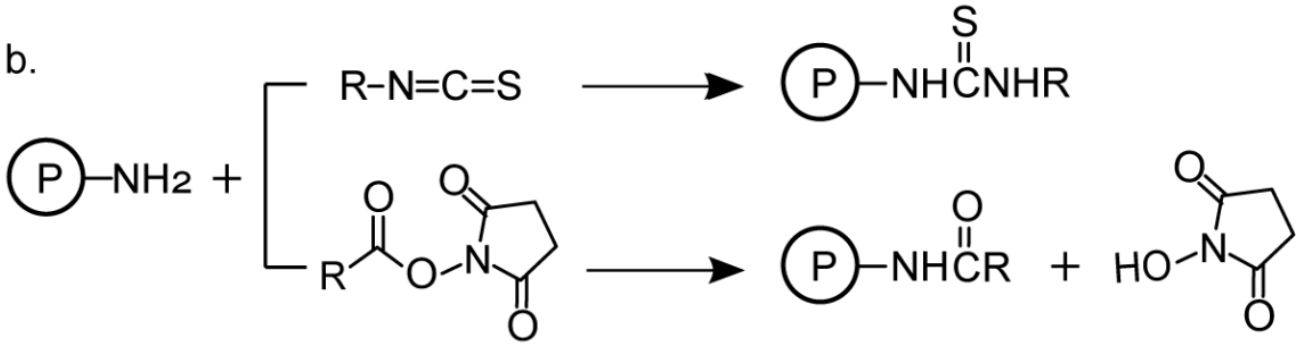

C.<smiles>c1ccccc1</smiles><smiles>N=C(N)N</smiles><smiles>[R]C(=O)C=O</smiles><smiles>[R]C1N=C(Nc2ccccc2)NC1=O</smiles>

d.<smiles>c1ccc(-c2c[nH]cn2)cc1</smiles><smiles>[R]C1CO1</smiles><smiles></smiles>

Figure 2. Common chemical modifications of proteins at cysteine (a), lysine (b), arginine (c), and histidine (d). P denotes protein; (a) shows the reaction of cysteine thiol group with either maleimides (top reaction) or with alkyl halides (bottom reaction) to form thioethers; (b) shows the reaction of lysine amino group with isothiocyanates to form thioureas (top) or with $\mathrm{N}$-hydroxyl succinimide esters to form carboxamides; (c) shows the reaction of arginine guanidinium group with $\alpha$-dicarbonyl compounds to form hydroimidazolones; (d) shows the reaction of histidine imidazole group with epoxides under alkaline condition. 


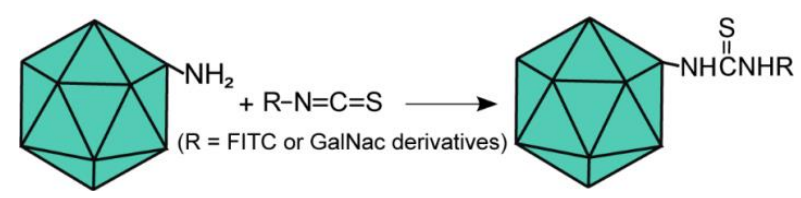

Figure 3. Modification of the AAV capsid Lys residues with isothiocyanate by Mevel et al. ${ }^{22}$ 


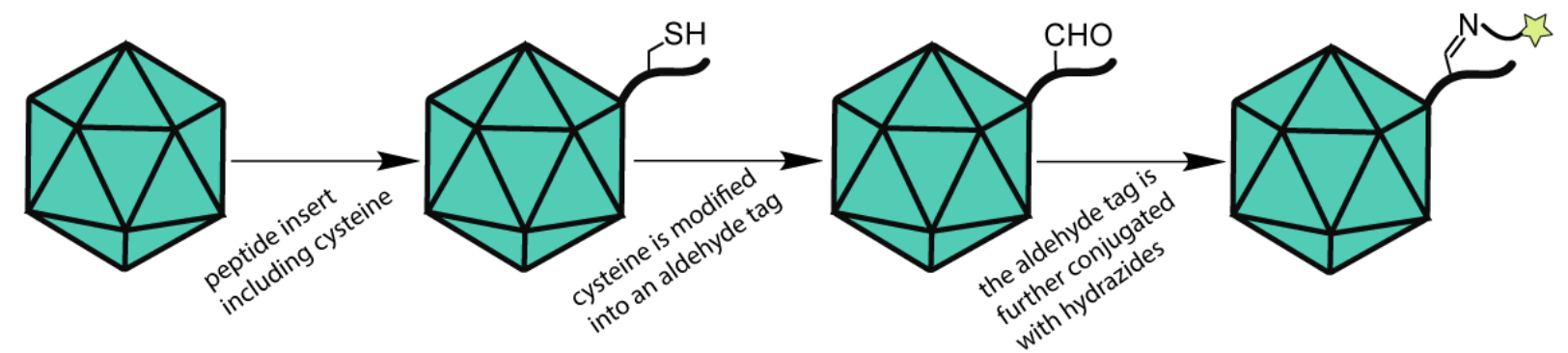

Figure 4. Schematic representation of Liu et al modification process. ${ }^{23}$ 


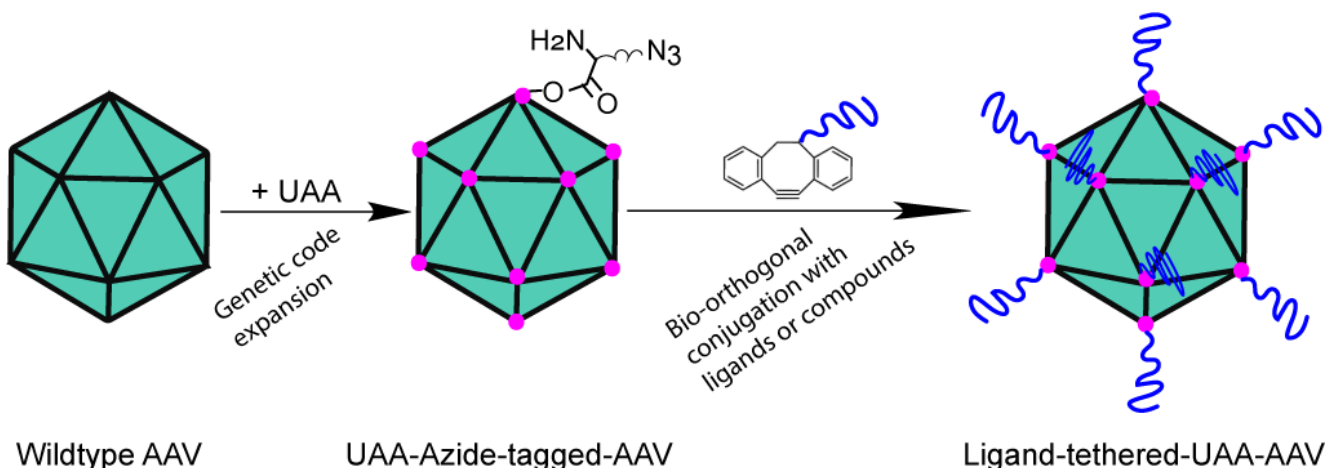

Figure 5. Representation of AAV modification via unnatural amino acid (UAA) approach. 